\title{
Lectures légitimes, lectures illégitimes
}

Se distinguer en lisant des littératures de l'imaginaire

Legitimate and Illegitimate Reading: Speculative Fiction Readers' Strategies of Distinction

\section{Élodie Hommel}

\section{(2) OpenEdition}

\section{Journals}

Édition électronique

URL : https://journals.openedition.org/bssg/579

DOI : $10.4000 /$ bssg. 579

ISSN : 2490-9424

Éditeur

Presses universitaires de Vincennes

Référence électronique

Élodie Hommel, «Lectures légitimes, lectures illégitimes », Biens Symboliques / Symbolic Goods [En ligne], 8| 2021, mis en ligne le 20 mai 2021, consulté le 23 juillet 2021. URL : http:// journals.openedition.org/bssg/579; DOl : https://doi.org/10.4000/bssg.579

Ce document a été généré automatiquement le 23 juillet 2021.

Biens Symboliques / Symbolic Goods 


\section{Lectures légitimes, lectures illégitimes}

Se distinguer en lisant des littératures de l'imaginaire

Legitimate and Illegitimate Reading: Speculative Fiction Readers' Strategies of

Distinction

Élodie Hommel

Fig. 1.

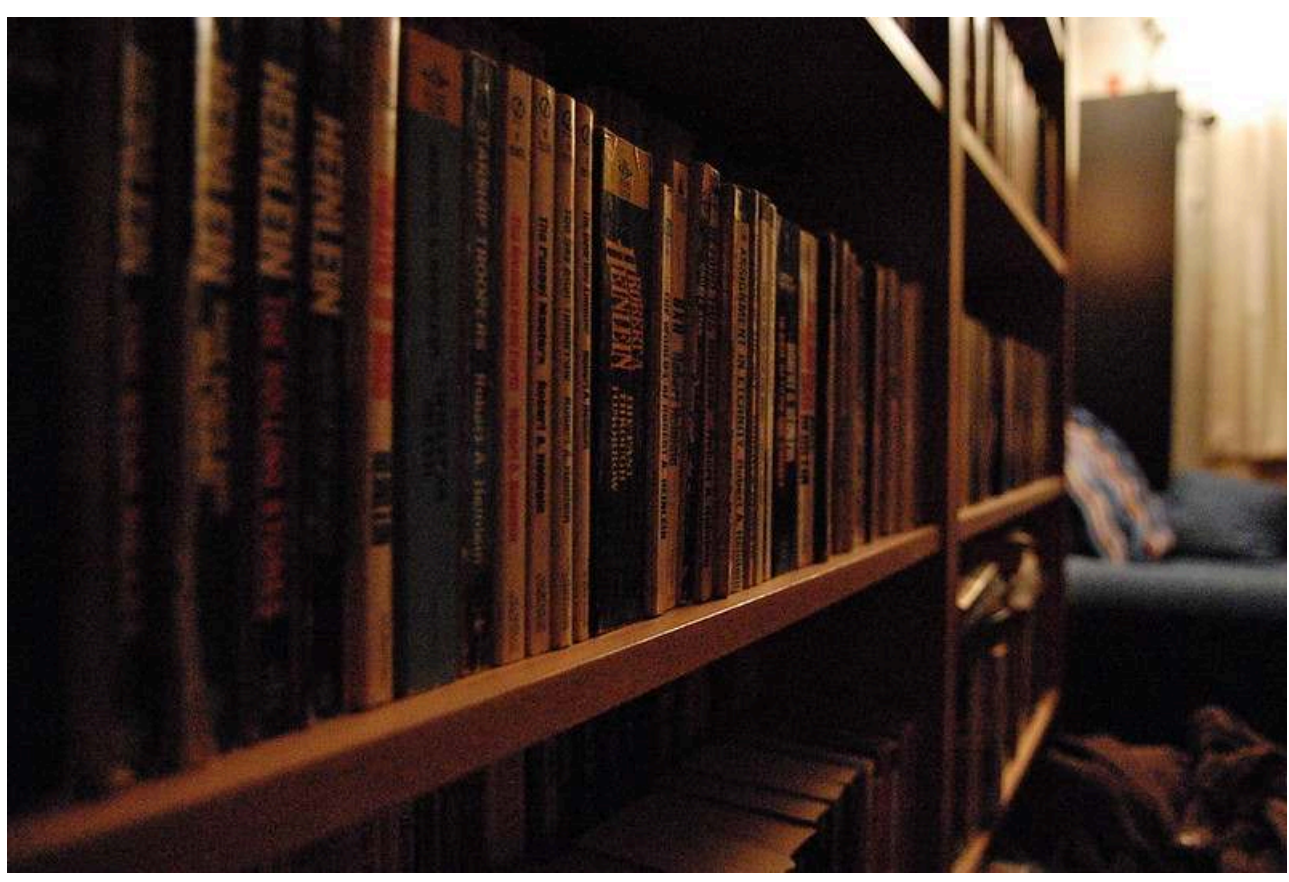

«Old-Time Science Fiction », par Ken Ficara

Source : Flickr 
1 Les littératures de l'imaginaire, qui englobent à la fois science-fiction et fantasy, regroupent des productions extrêmement variées, qui vont des œuvres "classiques", reconnues par les institutions culturelles dominantes, aux titres à gros tirages et au succès commercial. Associée au divertissement et à la jeunesse, cette catégorie éditoriale souffre dans son ensemble d'un manque de légitimité, comme en attestent les discours éditoriaux inquiets de l'image de ces genres. Une éditrice du Diable Vauvert déclare ainsi : «Nous avons intérêt à ne pas sortir nos auteurs dans une collection SF ${ }^{1}$. Ça les enferme » (Jakmakejian 2001).

2 Malgré ce caractère en partie illégitime, et malgré l'étiquette de littérature «populaire » qui leur est parfois attribuée, les littératures de l'imaginaire sont loin d'être rejetées par les publics à fort capital culturel : elles sont citées parmi les genres de romans lus le plus souvent par $31 \%$ des cadres et professions intellectuelles supérieures lisant des romans et par $28 \%$ des individus ayant un niveau d'étude correspondant à bac +2 ou bac $+3^{2}$ lisant des romans (Donnat 2009). La sociologie des publics et de la réception a par ailleurs montré à quel point l'association d'un type de production culturelle à un public était hasardeuse, aussi bien qu'à un mode de réception spécifique (Charpentier 2006) : on peut aussi bien lire Madame Bovary pour se divertir des rebondissements sentimentaux de la vie d'Emma que mener une analyse stylistique des pages d'Harry Potter. La lecture, peu importe sur quel genre elle porte, est susceptible de faire l'objet d'usages légitimes. En plus d'une ressource éthicopratique (Lahire 1993) elle devient alors ressource sociale : se dire lecteur ou lectrice constitue ainsi une forme de salut culturel (Mauger, Poliak \& Pudal 1999). Comment les lecteurs et lectrices s'accommodent-ils donc de la légitimité ambiguë des littératures de l'imaginaire? Comment transforment-ils cette lecture soupçonnée d'illégitimité en ressource culturelle et sociale? Quels usages en font-ils et quelles stratégies de distinction mettent-ils en place pour légitimer leurs pratiques de lecture?

3 Cet article, issu d'une thèse en sociologie sur les lectures de science-fiction et fantasy, se fonde sur une enquête qualitative menée entre 2014 et 2015, à partir d'observations d'évènements et de festivals dédiés au genre, d'une étude de l'offre éditoriale française contemporaine en littératures de l'imaginaire, et surtout d'entretiens avec des lecteurs et lectrices. Une quarantaine d'entretiens semi-directifs, d'une durée moyenne de trois heures, ont été réalisés auprès de jeunes adultes âgés de 20 à 35 ans, présentant aussi bien des lectures occasionnelles qu'assidues, exclusives au genre ou plus variées. L'approche adoptée a veillé à la variété des profils sociaux retenus, en termes de genre, de catégories socio-professionnelles, d'origine sociale, de lieu d'habitation et de parcours culturels, scolaires et universitaires.

4 Si les lectures des jeunes adultes interrogés peuvent faire l'objet de jugements de légitimité de la part de leur entourage (parents, professeure's ou pairs), nous verrons que ceux-ci ne constituent pas forcément des freins à la lecture. Les littératures de l'imaginaire présentent en effet une hiérarchie de légitimité interne, qui permet à celles et ceux qui en maitrisent les codes de se distinguer par leurs choix lectoraux. De véritables stratégies de distinction se mettent ainsi en place chez les lecteurs et lectrices, en particulier chez celles et ceux qui possèdent un fort capital culturel: privilégier les titres les plus littéraires, lire le texte en version originale, adopter des modes de lecture savants.

5 Mais le caractère en partie illégitime des littératures de l'imaginaire permet également à des lecteurs et lectrices culturellement moins bien dotée'es d'aborder la lecture via des 
textes moins « intimidants » que ceux de la culture classique. C'est alors le fait même de lire, peu importe le genre, qui devient distinctif et constitue une ressource sociale permettant de déjouer en partie les assignations statutaires.

\section{Jugements de légitimité : des freins à la lecture?}

\subsection{Inquiétudes parentales et bonne volonté culturelle}

6 Un certain nombre de jugements portés sur les choix de lecture des jeunes adultes interrogés apparaissent dans les entretiens. Les lecteurs et lectrices rapportent des discours critiques, souvent entendus pendant l'adolescence, mais qui peuvent persister au moment de l'enquête, présentant les littératures de l'imaginaire comme infantiles, pas assez sérieuses, ou encore trop éloignées de la réalité. Ces jugements émanent aussi bien des parents, que de l'institution scolaire ou des pairs, et pourraient constituer des freins à la lecture, comme c'est le cas chez les jeunes lecteurs et lectrices de mangas qui font face à de véritables « conflits de légitimité » (Détrez \& Vanhée 2012 : 237). Pourtant chez ces jeunes adultes, l'influence actuelle des prescriptions verticales semble moins forte que chez les adolescente-s interrogé'e's par Christine Détrez et Olivier Vanhée. En effet, avec l'entrée dans l'âge adulte, le souvenir des jugements scolaires et les opinions parentales exercent une pression moindre, et le départ du domicile familial permet également d'échapper au regard des parents sur les lectures effectuées. Ces critiques légitimistes des lectures n'ont pour autant pas été absentes du parcours des lecteurs et lectrices interrogés, comme l'évoque Marc :

«Ils auraient préféré que je lise autre chose. Que je sois plus dans l'optique, je sais pas... moins dans l'optique rêverie quoi. Plus dans lire des livres qui vont m'aider pour mes études plus tard, pour trouver un boulot, pour trouver... de toute façon quand j'étais au collège lycée, c'était vraiment dans l'optique "tu réussis tes études, tu fais tout pour réussir tes études", [...] Eux c'était les études, avoir un travail... gagner de l'argent. Moi, à quatorze ans, enfin quatorze quinze ans, je m'en foutais quoi. » Marc, 29 ans, sans emploi, niveau terminale ${ }^{3}$, père agent caténaire SNCF et chef d'équipe, mère sans profession.

$7 \quad$ Les critiques parentales pointent ainsi le caractère infantile supposé ( « c'est pour les enfants ») ou le manque de légitimité (" tu devrais lire autre chose ») du genre. Dans le cas de Marc, ce qui ressemble à une bonne volonté culturelle caractéristique des classes moyennes (Bourdieu 1979: 365) transparaît sous la forme d'inquiétudes liées à la réussite scolaire. Dans cette famille populaire, le rapport à l'école et la morale éducative (Buisson-Fenet \& Landrier 2008) mise en œuvre par les parents semblent relever d'une confiance en la méritocratie, qui permettrait à ceux qui s'en donnent les moyens de s'extraire de leur milieu d'origine et d'accéder à de nouvelles opportunités sociales et professionnelles ${ }^{4}$. Ainsi, les difficultés scolaires de Marc sont mises en relation par ses parents avec des choix de lecture jugés scolairement peu rentables.

La bonne volonté culturelle prend pourtant une forme différente chez les parents d'une autre partie des lecteurs et lectrices interrogés, principalement de bon'ne's élèves, dont les lectures n'ont jamais été remises en cause, mais plutôt encouragées (« Tant que je lis, ils sont contents »). Les bonnes notes à l'école, vues comme gages de sérieux et de capacité à se conformer aux prescriptions scolaires, autorisent à ces jeunes la liberté de lire ce qu'ils veulent, y compris des romans moins légitimes, à titre de détente. Le 
support du livre, culturellement plus légitime que d'autres objets culturels, compense sans doute ici l'illégitimité du genre.

Dans notre enquête, cette attitude parentale ouverte se retrouve aussi bien dans les classes populaires que supérieures, bien que la bonne volonté culturelle soit plutôt associée par Bourdieu aux classes moyennes, et bien que la posture consistant à s'autoriser des consommations culturelles peu légitimes à condition d'avoir par ailleurs démontré son aptitude à des pratiques plus légitimes rappelle plutôt l'éclectisme culturel des classes supérieures (Coulangeon 2004). La lecture de romans, même jugés peu sérieux, reste ainsi souvent un bastion culturel légitime et favorisé dans les discours parentaux, qui concentrent leurs critiques et leurs inquiétudes sur d'autres supports, comme le jeu vidéo ou le jeu de rôle, en lien avec les paniques médiatiques des années 2000 autour de ces pratiques ${ }^{5}$.

« Avec ma mère oui, à ce niveau-là... Quand je lui ai dit que je faisais du jeu de rôle, elle m'a demandé si j'étais suicidaire ou ce genre de choses, quoi ! Tu sais, les trucs que tu, en milieu rural que tu, qui sont ressassés par les médias, les légendes urbaines et tout, donc... Tous les rôlistes sont des suicidaires adorateurs de Satan, c'est bien connu! (ton ironique).» Julien, 25 ans, assistant d'éducation et pigiste, classe préparatoire scientifique ${ }^{6}$, master $d^{\prime}$ histoire, master métiers du livre, père ouvrier, mère en recherche d'emploi.

\subsection{Mésestime de l'institution scolaire}

10 À côté des jugements parentaux, nombreux:ses sont également les enquêtée's qui disent avoir subi les regards désapprobateurs de leurs professeur.e's. L'enthousiasme avec lequel lecteurs et lectrices évoquent les membres du corps enseignant ayant accueilli favorablement leur intérêt pour le genre dit en creux la rareté de ces postures professorales :

«Ce qui m'a fait plaisir, c'est que lundi, en cours de dissertation on a un prof qui a cité Tolkien, préface du Seigneur des Anneaux. Ah ! ça fait plaisir ! Ah ! ça fait plaisir (rires). » Marlène, 23 ans, étudiante en préparation à l'agrégation ${ }^{7}$ de grammaire, master de lettres classiques, père kinésithérapeute, mère cadre infirmière supérieure en maison de retraite.

11 La réception des travaux universitaires (exposés, mémoires ou même thèse de doctorat) portant sur les littératures de l'imaginaire en dit long sur la légitimité variable du genre, selon les disciplines (Marie-Claire, qui a étudié en école de commerce après une classe préparatoire, évoque ainsi une opposition entre cours de littérature anglophone et cours de littérature française), les institutions, ou encore les courants de recherche. Ainsi, Anaïs a pu réaliser un exposé sur un roman de fantasy en licence de lettres, Esther un devoir de modélisation sur la création du monde chez Robin Hobb en master de géographie, Philippe un mémoire d'études anglophones sur Dune et Perrine un mémoire sur les systèmes politiques dans la science-fiction. Pourtant, à côté de ces expériences réussies de transpositions des goûts lectoraux au monde universitaire, Perrine et Esther ont été découragées de poursuivre leurs recherches sur ce thème en doctorat. Perrine interprète le refus qui lui a été opposé comme une conséquence des restrictions budgétaires auxquelles l'université française est soumise, dissuadant le financement de thèses hors des champs supposément "porteurs" définis par l'administration de la recherche. Esther explique également son expérience par des facteurs liés aux politiques de recherche: avec le recul, elle réalise ne pas s'être adressée à des interlocuteurs inscrits dans le bon courant de recherche ${ }^{8}$ pour faire 
valider son projet. Si les littératures de l'imaginaire se sont fait une place dans les institutions universitaires, comme en témoigne la création de laboratoires de recherche (le Centre d'études et de recherches sur les littératures de l'imaginaire de l'Université Paris XII-Créteil ou le Centre de recherches littéraires sur l'imaginaire et la didactique de l'Université d'Artois), de revues scientifiques (Féeries depuis 2003, Res Futurae depuis 2012), de séminaires ou de colloques qui leur sont consacrés, cette reconnaissance est encore loin d'être générale.

\section{Une hiérarchie de légitimité interne au genre}

12 Si les jugements parentaux, professoraux ou médiatiques qui présentent les littératures de l'imaginaire comme un genre «mineur » appréhendent souvent celui-ci comme un tout, il existe pourtant une hiérarchie de légitimité interne au genre : ce sont les titres les plus commerciaux, ou encore destinés à la jeunesse, qui concentrent l'essentiel des critiques.

13 L'intitulé "littératures de l'imaginaire " regroupe en effet un ensemble foisonnant et pluriel de productions, des titres à gros tirages aux récits primés, qui peuvent relever de la science-fiction, de la fantasy ou de l'entre-deux. Ces différentes catégorisations font l'objet de nombreux débats et prises de positions, parfois virulentes, parmi leurs promoteurrrice's - auteurrrice's, éditeurrrice's et critiques littéraires (Torres 1997). En particulier, la définition de la science-fiction, la délimitation de ses frontières et ses liens avec la fantasy sont à l'origine de plusieurs lignes de clivage.

D'un côté, celles et ceux qui mettent l'accent sur le mot "science» dans "sciencefiction ", représentés en France par l'auteur et éditeur Gérard Klein, considèrent ainsi que le genre doit faire appel aux sciences "dures", et proposer des explications rationnelles ou réalistes aux phénomènes qu'il met en scène. Cette vision " puriste » de la science-fiction est la plus restrictive : elle exclut de fait tous les récits hybrides, faisant appel au merveilleux, qui s'écartent de la logique rationnelle. D'autres adoptent la définition du critique littéraire et universitaire Darko Suvin, en vertu de laquelle la science-fiction serait avant tout une projection dans un espace ou une temporalité différente, avec une composante littéraire ou philosophique (Suvin 1977). Le réalisme y importe moins, l'essentiel étant de mener une réflexion sur l'humain ou le rapport à l'autre (avec la figure de l'extraterrestre par exemple), réflexion rendue possible par ce déplacement. Ce courant est ainsi ouvert à tous types d'autres mondes, qu'ils relèvent de la projection futuriste ou du merveilleux. Il peut donc inclure des récits hybrides, ou même de pure fantasy.

15 À côté de ces enjeux de définition, propres à la science-fiction et aux littératures de l'imaginaire, on retrouve des oppositions plus classiques au sein du champ littéraire, entre pôle de production restreinte et pôle de production commerciale ${ }^{9}$ (Bourdieu 1991). La hiérarchie de légitimité interne au genre s'appuie donc à la fois sur des critères qui lui sont spécifiques et sur des critères littéraires plus généraux. L'offre éditoriale contemporaine de littératures de l'imaginaire reflète ces différents clivages, avec des politiques éditoriales qui s'opposent, entre logiques légitimistes et logiques commerciales, intégration ou exclusion de la fantasy, comme l'illustre le schéma cidessous ${ }^{10}$. 
Fig. 2.

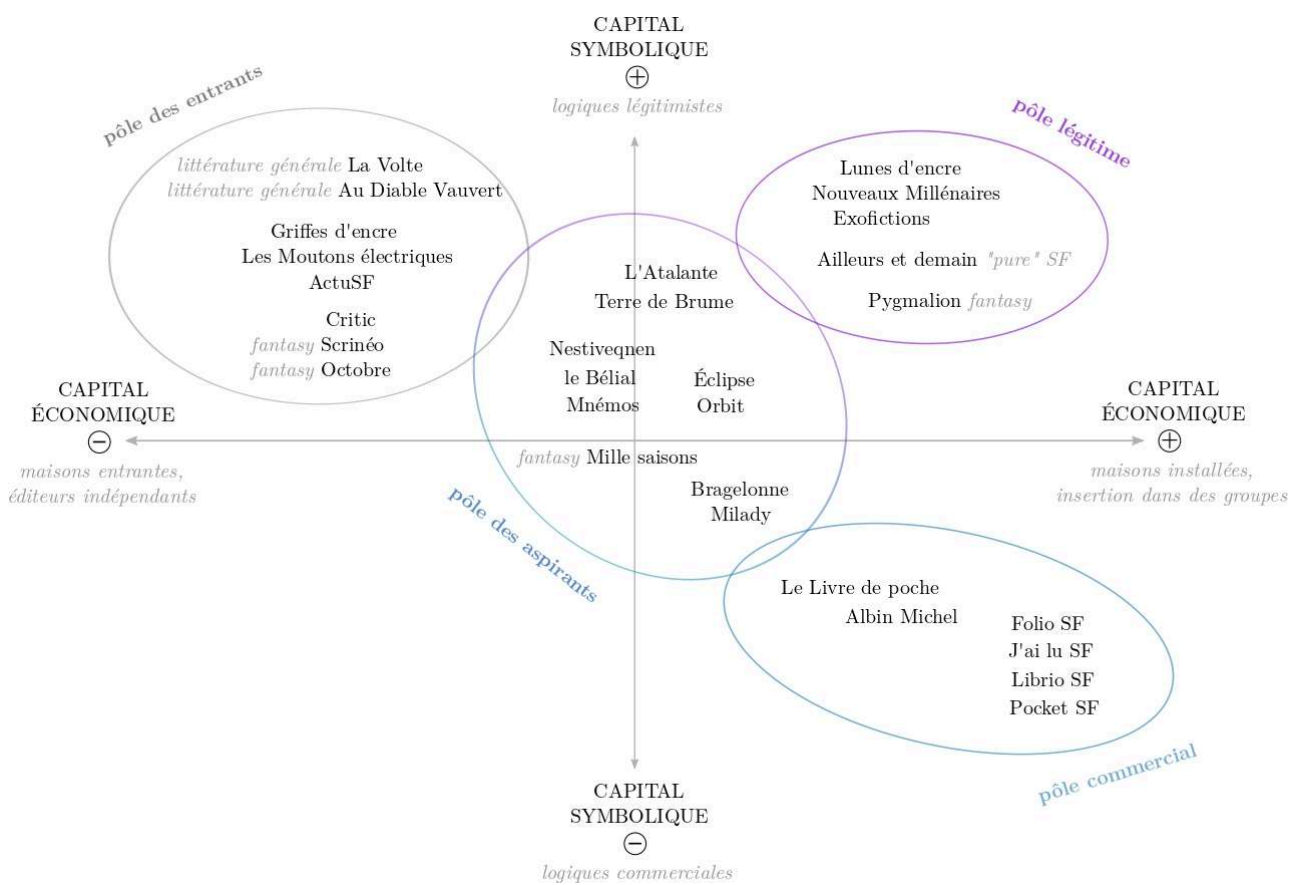

Cartographie de l'offre éditoriale française contemporaine en littératures de l'imaginaire

Source : Élodie Hommel (2017) lecteurs et lectrices interrogée's. Le recours aux critères spécifiques au genre, et notamment à l'évaluation du caractère rationnel ou scientifique des titres, est principalement le fait des lecteurs et lectrices qui ont suivi des études scientifiques ou travaillent dans le domaine des sciences et techniques. Ceux et celles-ci sont particulièrement attentifve's aux incohérences scientifiques présentes dans les romans. Leurs agacements vis-à-vis des exagérations, raccourcis ou invraisemblances des scénarios sont d'autant plus vifs lorsqu'il s'agit de leur domaine de spécialité, à l'instar de Laura, biologiste, face aux questions de génétique, ou d'olivier, physicien, face aux inquiétudes générées par les accélérateurs de particules. En plus de leur posture d'experts dans leur domaine, leur appréhension des mondes fictionnels est marquée par la démarche et le vocabulaire scientifiques: il s'agit d'accepter des " axiomes », d'imaginer leurs « implications », de " rester cohérent ».

La prise en compte des critères de hiérarchisation littéraires par les lecteurs et lectrices varie selon leur intériorisation des normes culturelles savantes. L'effort fourni au cours de la lecture constitue un premier critère de classement, entre titres faciles ou difficiles à lire. La mobilisation de ce critère se fonde avant tout sur l'expérience de lecture : nul besoin d'avoir suivi des études littéraires pour distinguer les livres lus facilement de ceux qui posent plus de difficultés. Cette expérience est individuelle : des textes jugés faciles par les uns peuvent sembler difficiles aux autres, selon leur aisance face à l'écrit. Mais le classement des lectures selon leur niveau de difficulté apparaît chez la quasitotalité des personnes interrogées, même si l'endroit où placer la limite entre facile et difficile varie individuellement, selon les compétences scolaires et l'intensité des pratiques de lecture. Les titres "faciles " sont souvent associés dans les discours des lecteurs et lectrices à la littérature pour adolescent·es, et considérés comme de 
moindre qualité, par opposition aux textes les plus «difficiles » qui requièrent de la concentration.

«Il y en a qui sont plus voilà, ben je dirais plus légers, comme Harry Potter ou Hunger games, voilà c'est des trucs sympas, c'est des univers magiques, mais on va dire faciles, alors que 1984 ou Philip K. Dick ou des choses comme ça, c'est beaucoup plus sombre, beaucoup plus adulte, et quand même plus compliqué, et c'est souvent tu lis entre les lignes pour comprendre ce qu'il veut dire, l'auteur. » Anne, 23 ans, webmaster et community manager, licence information communication et formation webmaster, père ingénieur et directeur d'école d'informatique, mère conseillère Pôle emploi.

Parallèlement à la difficulté de lecture, le caractère «commercial », "populaire » ou encore "mainstream ${ }^{11}$ » des titres constitue un autre critère de classement largement répandu. Un certain rejet des titres commerciaux, considérés comme des productions plus stéréotypées, "selon un processus classique qui consiste à faire de la dénégation de l'économique un signe de qualité » (Le Guern 2002 : 32), est présent chez la plupart des jeunes adultes interrogés. Ainsi, pour Thibault, le succès d'une saga ou d'un sousgenre annonce nécessairement une perte de qualité, la logique commerciale visant à rentabiliser une série à succès prenant le pas sur la logique artistique ou littéraire ( «si ça devient quelque chose à la mode, qui dit à la mode, ça devient un marché, et généralement, quand ça devient trop commercial, ben il va y avoir un petit peu de perte en ligne »). Mais cette hiérarchisation «esthète» des titres, opposant productions commerciales et textes de plus grande qualité littéraire, ainsi que la prise de recul par rapport aux conditions de production des titres, qui attestent de l'intériorisation des normes de légitimité savantes, sont surtout le fait des lecteurs et lectrices à fort capital culturel, hérité ou acquis.

« Il y a une grosse production, fin il y a eu, et il y a encore une grosse production, qui était une production à bas coût, pour un lectorat qui lisait ça rapidement, de manière, pour vraiment juste de la distraction pure, comme du bouquin de gare en fait, donc il y a eu une grosse production, mais il y en a des bons là-dedans je dis pas, mais il y a eu une grosse partie de, de production qui était de la production de commande, des auteurs qui faisaient voilà des productions de commande, donc qui écrivaient vite, avec des histoires simplistes qui se répétaient, des bouquins qui, dont la plupart sont tombés dans l'oubli, mais oui il y a une grosse partie de la production de SF qui est à la fois très facile à lire, et... voilà, écrite simplement. » Perrine, 31 ans, formatrice en savoirs généraux, master de sociologie politique, père chercheur en physique, mère employée.

19 La saga Twilight de Stephenie Meyer apparaît dans ces discours comme le repoussoir ultime, cumulant tous les stigmates : c'est un récit destiné à la jeunesse, qui se lit facilement, a rencontré un succès commercial et porte sur une thématique sentimentale. Si une partie des lecteurs et lectrices "avouent", selon leurs propres termes, l'avoir lue, elle ne trouve pas pour autant grâce à leurs yeux, en tout cas pas en situation d'entretien. À l'opposé du spectre de légitimité, certains titres sont mis en avant pour leurs qualités littéraires, voire élevés au rang de « classiques » du genre. Les critères de jugement d'ordre littéraire (qualité du style, richesse du vocabulaire ou complexité de la construction narrative) sont eux aussi le fait des individus issus de milieux sociaux favorisés, à fort capital culturel, comme Mathilde, et en particulier de celles et ceux qui ont suivi des études littéraires, et intégré la hiérarchie légitimiste des biens culturels.

"C'est vrai que, dans ce que je vais lire dans la SF et la fantasy, il y a quand même ce goût, tu vois, pour la littérature classique et pour la belle écriture qui reste, tu vois, 
je trouve, c'est peut-être pour ça que j'ai plus d'attachement à des gens comme Asimov et $\mathrm{K}$. Dick en SF, qu'à d'autres écritures plus modernes, je pense que j'ai un attachement au style littéraire." Mathilde, 29 ans, étudiante en master de pharmacie industrielle et chargée de recherches cliniques, études de sage-femme, licence de biologie, père médecin généraliste, mère médecin neurologue.

Les propos de Perrine, qui évoque «les romans qui sont presque [...] classés dans la littérature générale en fait, qui ont changé de rayon. Qui ont eu une sorte de promotion", témoignent non seulement de la maîtrise des normes littéraires qui placent certains titres de littératures de l'imaginaire au-dessus des autres, mais aussi de la façon dont cette hiérarchie s'articule avec celle des genres littéraires entre eux, et de la connaissance du phénomène $d^{\prime}$ ' extraction » du genre de certains titres qui sont alors admis au rang de la littérature générale (Klein 1977).

\section{Des stratégies de distinction}

\subsection{Le « fan », une figure repoussoir}

La connaissance et la maîtrise de la hiérarchie interne aux littératures de l'imaginaire permettent aux lecteurs et lectrices dotés de capitaux culturels (notamment familiaux ou scolaires) de mettre en place de véritables stratégies de distinction (Bourdieu 1979). Il s'agit ici de se différencier du lecteur " de base », du « mauvais lecteur ", voire d'une figure stéréotypée du "fan ", celui qui vivrait sa lecture "sur le mode d'une adhésion passionnelle, voire pathologique » (Le Guern, 2002 : 24). Dans le cas des littératures de l'imaginaire, comme pour d'autres objets culturels perçus comme peu légitimes, l'illégitimité de ce rapport passionnel à l'œuvre se double de l'illégitimité de l'œuvre elle-même. En outre, dans un contexte où l'éclectisme culturel peut être analysé comme un nouveau ressort de distinction (Coulangeon 2003), l'exclusivité d'une pratique, caractéristique de la figure $\mathrm{du}$ "fan», autrement dit le désintérêt pour d'autres pratiques, devient également facteur d'illégitimité.

La mise à distance de la figure péjorative du «fan", et notamment de ses avatars les plus dévalorisés (Le Bart 2004: 301-302), par les lecteurs et lectrices en quête de distinction, passe par la stigmatisation des attitudes considérées comme les plus illégitimes : collectionner des produits dérivés, faire dédicacer ses livres, se projeter sans recul dans le monde représenté, se déguiser en personnages. Les aspects les moins légitimes de la passion sont souvent associés à la jeunesse, à une immaturité, voire à une certaine naïveté1 ${ }^{2}$, qui est celle de l'enfance ou de l'adolescence. Lecteurs et lectrices, en particulier dotée'es de capitaux culturels, soulignent alors leur distance aux plus jeunes, mais aussi à leurs propres pratiques de jeunesse, dans une distinction « de soi à soi » (Lahire $2004: 30$ ), qui les porte à mettre en évidence les modes de lecture plus légitimes qui sont désormais les leurs. Les postures de fans valorisées, comme l'approche esthétique, la création et surtout l'expertise sont ainsi mises en avant, en situation d'entretien, mais aussi plus généralement dans leur rapport aux autres lecteurs et lectrices.

L'étiquette «fan » peut ainsi être revendiquée en tant que figure d'expertise, comme le fait Luc, mais à condition de bien la distinguer du fan «de base " (que Luc oppose au "grand fan", auquel il s'identifie). L'expert s'inscrit dans une démarche encyclopédique, à l'instar de Thierry quand il se dit " capable de lire de la chick-lit ${ }^{13}$ » : s'imposer de lire ces productions peu reconnues fait alors partie d'une démarche 
experte, visant à la connaissance exhaustive du genre. Si l'adoption de postures culturellement valorisées permet aux plus assurée's de retourner le stigmate et d'assumer l'étiquette de fan, pour une grande partie des lecteurs et lectrices interrogée's, le terme reste connoté trop négativement. Ils et elles expriment alors des réticences à l'employer pour parler d'eux/elles-mêmes, lui préférant des mots moins marqués, comme "amateurrice», "passionnéee ou encore "connaisseur-se ». Cet idéal-type du/de la "fan», qui serait un·e mauvais·e lecteurrrice, n'a pourtant pas été rencontré en tant que tel dans notre enquête, où toutes les personnes interrogées ont un usage réflexif minimal de leurs lectures ${ }^{14}$.

\subsection{Des choix et des modes de lecture distinctifs}

La distinction passe d'abord par le choix des titres lus, l'enjeu étant de ne pas lire " comme tout le monde». Les lecteurs et lectrices à fort capital culturel sont ainsi nombreux'ses, comme Sébastien, à mettre en avant les titres peu connus qu'ils et elles ont pu lire, à souligner leur recherche d'originalité dans leurs choix de lecture.

«Je trouve la SF française super créative! [...] les auteurs français sortent des sentiers battus. Oui. Et essayent de sortir un petit peu des schémas classiques, ou alors quand ils les utilisent... parce que c'est vrai que Sylvie Lainé, dans son recueil L'opéra de Shaya, je crois que, dans à peu près toutes les nouvelles, c'est plus ou moins explicite qu'il y a un ordre galactique, qui est établi. Mais... ce n'est pas le sujet, en fait, le sujet, c'est quelque part, un individu à un moment et je trouve ça hyper intéressant.» Sébastien, 26 ans, chargé de communication, BTS ${ }^{15}$ informatique, maîtrise ${ }^{16}$ communication et évènementiel, père ingénieur en informatique, mère assistante de direction.

La lecture de titres réputés difficiles, peu accessibles, longs, ou nécessitant un haut niveau de concentration constitue également un motif de fierté pour les enquêtée's qui en sont venuees à bout, et peuvent ainsi se distinguer de la masse des lecteurs et lectrices " ordinaires ", en situation d'entretien mais aussi de façon plus informelle, au cours de discussions entre ami-e's ou en famille, où certain'e-s adoptent des postures d'expertise sur le genre ${ }^{17}$. Lire des récits dans une langue ancienne ou étrangère participe aussi à ces phénomènes de distinction, à la manière des étudiants de classe préparatoire littéraire qui «s'autorisent" la lecture de romans peu légitimes à condition de les lire en version originale (Maridet $2016: 449-450$ ). Thibault évoque ainsi les nouvelles médiévales merveilleuses qu'il a lues en français médiéval, Maxime le vocabulaire mathématique aride d'Isaac Asimov dans Fondation, tandis qu'Amaury affirme avoir étudié Le Seigneur des anneaux en «vieil anglais $»^{18}$. L'animosité qu'il exprime à l'égard de la version originale (et le conseil donné à l'enquêtrice de ne pas la lire) est aussi une manière de valoriser sa propre lecture du texte, inaccessible au plus grand nombre.

"Alors Le Seigneur des anneaux, je l'ai étudié en vieil anglais, mon dieu, c'est horrible, c'est horrible, comment te dégoûter à tout jamais de lire ce bouquin (rire) c'est affreux. Pour le coup, tu vois, la version française est la plus moderne, la plus facile à lire, c'est... Ne surtout pas lire la version originale. " Amaury, 30 ans, sans emploi et gestionnaire bénévole dans une boutique de jeux, licence $\operatorname{LLCE}^{19}$ anglais, père employé d'imprimerie, mère hôtesse d'accueil.

26 À l'opposé, les titres considérés comme mauvais par ces lecteurs et lectrices cultivé·e·s sont rejetés, voire décriés. Fréquemment, les jeunes adultes concernés affirment qu'ils et elles sont incapables de lire ce type de productions, comme si la hiérarchie des biens 
culturels légitimes, le " dégoût du goût des autres ", était incorporée au point d'affecter leurs capacités de lecture (alors que ces mêmes lecteurs et lectrices n'hésitent pas à mettre en valeur le fait qu'ils et elles aient dû «se forcer " à finir des titres difficiles mais littérairement reconnus). L'autre dont il s'agit de se distinguer, c'est alors le lecteur-consommateur, qui ne dispose pas des compétences lectorales suffisantes pour séparer le bon grain de l'ivraie et qui se satisfait des productions identifiées comme commerciales par les lecteurs et lectrices plus avisée's. Cette identification tient aussi bien de la compétence d'étiquetage des titres et collections en fonction de leur degré de prestige $^{20}$, que de l'expérience concrète de lecture, qui achoppe sur un registre trop pauvre ou sur la répétition de schèmes narratifs éculés. La qualité insuffisante du style ou du travail scénaristique est ainsi susceptible de faire obstacle au plaisir de lecture.

«Il y a des bouquins comme ça, ça m'est arrivé d'aller acheter des bouquins dans les kiosques à deux euros le bouquin, principalement de l'heroic fantasy épouvante, et euh... sur dix bouquins achetés, il y en a huit que j'ai laissés de côté, j'ai arrêté de lire.» Mégane, 31 ans, galeriste, master communication et gestion tourisme international, père ouvrier d'État, mère infirmière.

Celles et ceux qui lisent tout de même ces titres adoptent une posture critique, soulignant la distance prise avec un texte dont la lecture pourrait les stigmatiser. Marie-Claire raconte ainsi avoir lu Twilight en secret alors qu'elle était en classe préparatoire.

La distinction peut aussi passer par la connaissance exhaustive d'une œuvre, qui permet d'en comprendre les différentes ramifications, et de se distinguer de ceux qui n'ont lu «que » les titres les plus connus, et n'en tirent ainsi pas tout le sel. C'est le cas de Thibault, qui se flatte de la compréhension accrue du Seigneur des anneaux que lui apporte sa lecture du Silmarillion ${ }^{21}$ de Tolkien, ou de Luc avec l'univers Marvel, qu'il connait via les comics et non uniquement par les adaptations cinématographiques.

"C'est vraiment, vraiment la Genèse. En fait Le Seigneur des anneaux dans Le Silmarillion, c'est cinq pages, c'est pas grand-chose, donc effectivement, je sais pas, il y a peut-être un côté un peu élitiste qui fait que t'as lu le Le Silmarillion, tu sais dix fois plus de choses sur Le Seigneur des anneaux, que les gens qui ont juste lu Le Seigneur des anneaux, ou qui ont juste vu les films, ben ils savent pas, ils comprennent pas...» Thibault, 32 ans, responsable communication et marketing, DUT $^{22}$ génie biologique et environnement, Deug ${ }^{23}$ sciences de la terre, école de commerce, père chef cuisinier, mère femme au foyer.

\section{Lectures et compétences savantes}

Au-delà du choix des titres lus, ce sont aussi les manières de lire qui sont distinctives. Il est ainsi possible de sublimer des titres peu légitimes en les appréhendant selon les normes de la culture savante, ou comme l'exprime Pierre Bourdieu « c'est la manière de consommer qui crée en tant que tel l'objet de la consommation et la délectation au second degré transforme les biens "vulgaires" livrés à la consommation commune, westerns, bandes dessinées, photos de famille, graffitis, en œuvres de culture distinguées et distinctives » (Bourdieu 1979 : 321). Ce sont donc également les façons de lire considérées comme peu légitimes qui sont rejetées par les lecteurs et lectrices à fort capital culturel, se livrant au cours de l'entretien à de véritables analyses littéraires, stylistiques ou encore philosophiques des romans lus, dans une attitude de 
distinction valorisant un mode de lecture élitiste par rapport aux lectures « ordinaires".

Si le plaisir est loin d'être absent des propos des lecteurs et lectrices interrogée's, c'est pourtant la lecture de divertissement, celle du « lire pour le plaisir » (Mauger, Poliak, Pudal 1999), qui concentre l'essentiel des critiques. Ce qui est critiqué n'est pas tant le fait de ressentir du plaisir en lisant que la recherche exclusive de ce plaisir dans la lecture, et l'immersion sans recul réflexif dans le monde fictionnel, au détriment d'autres usages de la lecture jugés plus légitimes (didactique, «de salut» ou " esthète »). Si, d'après Gérard Mauger, Claude Poliak et Bernard Pudal, la lecture esthète " pure » existe rarement en tant que telle, on peut également s'interroger sur l'existence d'une lecture de "pur divertissement", tant les enquêtes sur la lecture montrent que ces différents usages sociaux se combinent au fil d'une même lecture, dans des proportions variables selon les individus et les circonstances. L'évocation du mode de lecture esthète, dans une mise en scène de soi à visée distinctive, est en revanche fréquente parmi les lecteurs et lectrices interrogéee's.

« Il y a des lectrices comme moi, qui justement sont plutôt dans la réflexion et dans l'échange plutôt que dans l'immersion et que... Tout dépend en fait c'est pas vraiment le genre qui va influencer le style de lecteurs qu'il y aura en face, mais ça dépend aussi, c'est des lecteurs qui font des rencontres avec un genre particulier et que ces personnes qui sont à s'immerger dans un monde, ça serait pas un monde de fantasy elles auraient le même rapport à la lecture avec un autre bouquin donc... il faut pas diaboliser la fantasy et la SF... c'est le mode de lecture qu'il faut... on sent la prof de français là (rires).» Ophélie, 22 ans, professeur de français stagiaire, $1^{\mathrm{e}}$ année de Master $^{24}$ lettres modernes, classe préparatoire littéraire, père informaticien, mère infirmière.

31 Lecteurs et lectrices mettent alors en œuvre des compétences stylistiques et culturelles, acquises dans le cadre scolaire ou de façon autodidacte via des lectures intensives. Dans son travail sur les lectures des lycéens, Fanny Renard distingue notamment trois types de «lectures analytiques": la lecture-résumé, la lecture stylistique et la lecture comparative (Renard 2011 : 100-103), qu'on retrouve, dans des proportions variables, chez les lecteurs et lectrices de science-fiction et fantasy interrogéees.

\subsection{Comparer et contextualiser les textes}

Les jeunes adultes interrogée's se livrent toutes et tous, de manière plus ou moins développée, au jeu de la lecture comparative. Celle-ci va de la comparaison entre un roman et son adaptation filmique ou ludique, à la recherche de similitudes entre les romans lus (personnages, schémas narratifs, thématiques), en passant par des analyses comparatives étoffées entre plusieurs œuvres et le repérage de références culturelles ou littéraires plus larges. Dans ses analyses sur les «cultes médiatiques », Philippe Le Guern observe que le public tient souvent "un discours hyper-savant sur l'œuvre culte » (Le Guern 2002 : 23). De la même manière, lecteurs et lectrices de littératures de l'imaginaire mobilisent des références nombreuses et variées, issues de la sciencefiction et de la fantasy comme d'autres genres littéraires.

"John Keats c'est un poète anglais, début XIX et euh, et justement, il y a énormément de, il y a énormément de son univers dans, dans Hypérion, c'est pour ça qu'il y a énormément de poésie euh, une manière de voir le monde et de considérer la littérature et de multiplier la littérature et les poètes qui est très intéressante. » Gauthier, 26 ans, étudiant en master production et distribution cinéma, master 
stratégie des échanges culturels internationaux, père professeur d'histoiregéographie, mère psychologue scolaire. plus poussées et les références les plus abondantes sont le fait des lecteurs et lectrices les plus dotée's en capitaux culturels (car issu'e's de familles à fort capital culturel, ayant suivi des études supérieures littéraires ou grand·e's lecteurs et lectrices). Les références convoquées varient également selon la diversité des lectures des individus, soit exclusivement effectuées en science-fiction et fantasy, soit empruntées à d'autres genres, y compris à la littérature classique. Pour les lecteurs et lectrices à fort capital littéraire, la démarche dépasse la comparaison, et consiste plutôt à rechercher « les influences ", les titres qui «ont marqué » les auteurrice's des romans lus, dans une perspective plus proche de l'analyse littéraire, celle de l'identification critique des sources. présente chez les jeunes adultes interrogés. Ici encore, elle prend différentes formes selon l'investissement dans la lecture et le niveau de capital culturel. Plus de la moitié des lecteurs et lectrices déclarent se renseigner, au moins de temps en temps, sur la biographie ou la bibliographie des auteur.rice's lue's. Les moins investis se contentent de lire les notices bio-bibliographiques incluses dans les romans ou les pages Wikipédia correspondantes, tandis que d'autres se livrent à des recherches plus poussées, voire se lancent dans la lecture de biographies détaillées. Les connaissances ainsi acquises permettent de replacer les titres lus dans leur contexte de production (situation personnelle de l'auteurrrice, cadre historique et culturel de l'écriture).

Pour les jeunes adultes qui ont suivi des études littéraires, la lecture des préfaces devient un " réflexe ", qui complète cette démarche de contextualisation biographique et culturelle. Le goût de la recherche bibliographique relève des mêmes trajectoires. Plusieurs lecteurs et lectrices interrogéees ont ainsi eu l'occasion, dans le cadre de leur cursus universitaire ou à titre personnel, de lire des analyses critiques sur les littératures de l'imaginaire dans leur ensemble ou sur un titre en particulier.

\subsection{Une attention inégale à l'écriture}

36 Le rapport analytique aux textes peut aussi passer par la lecture stylistique: par l'attention à l'écriture et à la structure narrative des récits, par l'analyse littéraire des textes, celle-ci « rend possible la production d'un commentaire sur la construction du texte, la mise en évidence de ses effets sur un lecteur-étalon et l'élucidation des thématiques et points de vue sur le monde dont le texte est porteur » (Renard 2011: 101). Dans le cas des littératures de l'imaginaire, ce type de compétences analytiques peut être mobilisé, qu'il s'agisse d'étudier la construction narrative, le style du récit ou encore le système des personnages.

«Alors le style de Nancy Kress je la connaissais pas, alors j'ai beaucoup apprécié, en fait elle a un style assez brutal, avec des chapitres, enfin ça tombe de manière brutale quoi. On a l'impression que c'est coupé au cordeau, il y a rien qui est superflu. » Marina, 29 ans, documentaliste en santé publique, licence de biologie et DUT documentaliste, père technicien supérieur à France Télécom, mère vendeuse.

37 Ces compétences littéraires sont plus présentes chez les lecteurs et lectrices qui ont suivi des études en lettres et sciences humaines, ou auxquelless la famille leur a transmis un fort capital culturel, mais on les retrouve également chez les grandees 
lecteurs et lectrices, qui ont développé une familiarité avec les textes et leurs codes via leurs pratiques lectorales intenses. Bien que le vocabulaire d'analyse littéraire soit moins développé chez ces derniers, leurs propos font état d'une sensibilité au style et d'une capacité d'analyse éprouvée.

À côté des compétences d'analyse, c'est aussi une inégale intériorisation des hiérarchies littéraires scolaires et universitaires qui transparait dans l'attention portée au style et dans les critères de jugement des œuvres. Dans notre enquête, les effets du milieu social d'origine se font sentir, puisque les jugements selon des critères littéraires sont plus présents chez les individus issus de familles à fort capital culturel, mais ils se mêlent à ceux des études suivies et à l'intensité de la pratique lectorale. Ainsi, Nadia, agrégée de géographie, bien qu'issue d'une famille à faible capital culturel, a intériorisé les normes savantes à travers ses études supérieures poussées ( Je classe quand même la littérature comme une écriture qui devrait être un peu au-dessus ») tandis que Marc, également de classe populaire, mais qui n'a pas fait d'études et lit modérément, accorde peu d'importance à l'écriture ( «la façon dont il écrit, la façon dont c'est tourné... je m'en fous de ça, s'il m'a plu à moi »).

La relecture, qui peut par ailleurs jouer un rôle émotionnel chez les lecteurs et lectrices interrogée's, en les replongeant dans des univers familiers, se fait aussi parfois dans une perspective savante : elle permet d'approfondir l'analyse, d'étudier le texte plus consciencieusement que lors de la première lecture. Parmi les jeunes adultes interrogés à qui il arrive de relire des livres, nombreux'ses sont celles et ceux qui déclarent ainsi relire en prêtant attention aux détails ou à d'autres dimensions, ou avec un regard enrichi par des connaissances acquises entre temps.

«Le vent de feu là, oui oui, il y a quand même, en fait c'était un prof de philo à la base qui avait fait ça, donc il y a plein de références philosophiques tout au long de l'histoire, et puis en fait, j'ai redécouvert plus tard, moi-même en ayant fait de la philo et tout ça. Donc là oui, et là du coup c'est d'autant plus intéressant de le relire qu'avant. » Ophélie, 22 ans, professeur de français stagiaire, M1 lettres modernes, classe préparatoire littéraire, père informaticien, mère infirmière.

Les littératures de l'imaginaire, comme d'autres productions culturelles dont la légitimité n'est pas assurée (Collovald \& Neveu 2004 ; Détrez \& Vanhée 2012), se prêtent aussi bien aux lectures ordinaires qu'aux lectures savantes, confirmant qu'on ne peut se contenter d'associer un type de production culturelle à un type de public et de lecture. Contextualiser leurs lectures, saisir les jeux de références, analyser le style et la construction narrative, notamment en se prêtant à la relecture des textes : lecteurs et lectrices interrogée's mettent en œuvre toute une série de compétences littéraires, plus ou moins développées selon le capital culturel des individus, que celui-ci soit hérité, acquis via les études ou accumulé par des lectures nombreuses.

\section{La lecture comme ressource sociale et culturelle}

\subsection{Lire pour conserver un statut social}

41 La volonté de se distinguer et de sortir du lot n'est pas l'apanage des lecteurs et lectrices issuees de familles à fort capital culturel ou de ceux qui ont acquis un tel capital par des études supérieures littéraires. La lecture, de littératures de l'imaginaire comme d'autres genres littéraires, peut constituer une ressource culturelle pour des jeunes adultes des classes populaires, en particulier quand ils se trouvent dans des 
situations professionnelles précaires. Le fait de lire, peu importe quoi, constitue alors à lui seul un critère de distinction par rapport à d'autres individus, dont les pratiques culturelles sont considérées comme moins légitimes. Pouvoir se dire lecteur ou lectrice, malgré les circonstances, constitue ainsi en soi une forme de «salut culturel» (Mauger, Poliak, Pudal 1999).

C'est par exemple le cas de Marc. Après avoir arrêté ses études en terminale, sans passer le baccalauréat, il a enchaîné plusieurs contrats précaires en tant qu'employé ou ouvrier, ainsi que quelques années dans l'armée. Au moment de l'entretien, il se trouve dans une situation extrêmement précaire puisqu'il est au chômage et a été obligé de quitter son appartement. Il vit actuellement « en squat » et la lecture lui permet à la fois de s'évader de ses difficultés quotidiennes et de se distinguer des personnes qui vivent avec lui. Conserver une activité culturelle malgré le déclassement subi lui permet en effet de se différencier des autres «squatteurs », parfois sans domicile fixe depuis plusieurs années, et de mettre à distance la possibilité de ne pas réussir à s'en sortir lui-même. Continuer à lire et continuer à chercher du travail deviennent ainsi les deux faces d'un même combat visant à conserver une position sociale digne.

«Dans le milieu du squat non, ben après c'est des gens qui ont vraiment pas cette... la lecture c'est vraiment quelque chose qui leur passe au-dessus, enfin ils ont vraiment d'autres préoccupations, enfin il y en a qui ont vécu dans la rue depuis plusieurs années, il y en a qui ont, ben qui sont HS quoi, qui ont plus du tout la préoccupation d'avoir une distraction culturelle ou enfin la culture ça leur passe au-dessus quoi. Pour eux c'est manger, oublier là où ils sont. " Marc, 29 ans, sans emploi, niveau terminale, père agent caténaire SNCF et chef d'équipe, mère sans profession.

La situation est similaire pour Benjamin, conducteur de tramway : la lecture lui permet de se distinguer de ses collègues, dont les pratiques culturelles sont réduites, selon lui, au sport et à la radio. Là encore, la lecture rend possible une extraction d'un milieu social, ici le milieu professionnel, qui fait office de repoussoir, et qui s'oppose à un cercle amical fait de nombreux lecteurs et lectrices avec qui partager cette passion.

«Disons que quatre-vingt-cinq, quatre-vingt-dix pour cent de mes collègues c'est radio bière foot, donc c'est pas très, très... [...] C'est pas le même genre. J'ai deux trois collègues, avec qui ça passe bien, avec qui je peux en discuter ou quoi. Pour prendre un exemple, moi je lis là-dessus [désigne sa liseuse] au boulot, parce que ça tient dans ma poche, quand ils me... je peux pas avoir la paix deux minutes. Ah c'est bien, c'est quoi, c'est un Kindle, ça sert à quoi ? Ben c'est pour lire des bouquins... [...] C'est pas vraiment le public de littérature... » Benjamin, 30 ans, conducteur de tramway, niveau terminale, père chauffeur poids lourds, mère assistante familiale.

Dans les deux cas, ces volontés de distinction chez des jeunes adultes de milieu populaire peuvent avoir été nourries par un environnement familial où les mères lisaient ${ }^{25}$, mais aussi par les pairs, via des liens de sociabilité avec des individus issus de milieux plus dotés culturellement, à l'instar de l'ex-petite amie de Marc, ou des amis de Benjamin. Comme la lecture de romans policiers, la lecture de science-fiction et fantasy leur permet de "déjouer les assignations statutaires » (Collovald \& Neveu $2004: 247$ ), en conservant une dignité sociale par le maintien de pratiques culturelles légitimes dans une situation de précarité, ou de s'extraire d'un milieu jugé culturellement trop faible pour tendre vers une identité sociale plus valorisée, celle de lecteur ou lectrice. 


\subsection{Quand l'imaginaire se fait capital culturel}

45 constituer une porte d'entrée dans la lecture, aidant ainsi lecteurs et lectrices à se constituer un capital culturel. Julien raconte par exemple comment les romans de fantasy ont aidé les jeunes lycéens en difficulté qu'il surveille dans le cadre de son travail d'assistant d'éducation à découvrir la lecture, à y prendre plaisir, et à y trouver des ressources face à un quotidien difficile.

«Un jour il y en a un qui est venu, enfin lui il est venu me voir en me demandant... "Du coup j'ai appris à lire y'a deux ans, je lis pas très bien, j'aimerais bien lire un bouquin. Mais je veux lire un bouquin comme tu lis. " "C'est-à-dire, c'est-à-dire quoi un bouquin comme tu lis? " "Bah, bien! " "Ça veut dire quoi? " "Ben quand je te vois lire... T'es concentré, des fois tu souris, des fois tu fronces les sourcils, puis quand tu nous en parles, t'es toujours... Ça se voit que ça t'a plu et que t'es content de les avoir lus, tu passes un bon moment mais... Je m'emmerde tellement dans ma vie que je pense que ça me ferait du bien". Un gamin de 19 ans qui te dit ça, tu te dis, quand même sa vie... ouais. Et, lui s'est mis à lire du Neil Gaiman (rire) bon, il avait jamais lu de sa vie il a commencé un peu avec des livres on va dire ado, et il s'est mis à lire le reste, un autre s'est mis à lire du... Des gamins qui avaient rien à voir avec la littérature de l'imaginaire ou la culture du bouquin. L'autre s'est mis à lire du David Gemmel, il s'est tout enfilé, il y en a un autre qui s'est mis à lire Eddings il s'est tout enfilé pareil, en trois mois." Julien, 25 ans, assistant d'éducation et pigiste, classe préparatoire scientifique, master d'histoire, master métiers du livre, père ouvrier, mère en recherche d'emploi.

Son récit évoque une initiation aux littératures de l'imaginaire qui se fait par imitation, et non par inculcation, à la demande de l'élève, et non à l'initiative de l'adulte. La scène a lieu dans un cadre scolaire, mais décalé : en salle de permanence plutôt qu'en salle de classe, face au surveillant plutôt qu'au professeur. Bien que socialement improbable, la mise à la lecture est ici rendue possible par la mise en présence d'acteurs, que leur âge contribue peut-être à rapprocher, et par l'observation d'une pratique méconnue. Comme le souligne Michèle Petit, "pour nombre de jeunes qui se sentaient peu autorisés à s'aventurer dans la lecture d'œuvres littéraires, du fait de leur origine sociale, une ou plusieurs rencontres avec un enseignant - ou avec un bibliothécaire ont été décisives. Il ne s'agit pas forcément de relations régulières, suivies, sur le temps long, car même fugace, une conversation peut quelquefois infléchir le destin » (Petit $2002: 167$ ).

Par ailleurs, pour les individus qui lisent déjà, les littératures de l'imaginaire peuvent aider à apprivoiser des pans de la culture jugés intimidants : plusieurs personnes interrogées affirment ainsi avoir osé aborder des «classiques» par le biais de la science-fiction ou de la fantasy. La littérature classique, celle des prescriptions scolaires, jugées ennuyeuses ou difficiles, dans tous les cas "pas pour eux", est ainsi approchée de côté, via certains titres spécifiques qui s'apparentent aux littératures de l'imaginaire et où ces lecteurs et lectrices retrouvent ce qu'ils apprécient dans le genre, tout en se confrontant à un autre monde culturel. C'est le cas de Céline, grande lectrice de fantasy, issue d'un milieu favorisé (père médecin, mère pharmacienne) où on lit, mais où la culture dite « légitime » est absente : dans cette famille de scientifiques, la mère lit surtout de la science-fiction et des policiers, le frère de la fantasy, et seule la sœur cadette (qui étudie la psychologie à l'université) lit « des biographies ». Elle décrit la découverte inattendue d'un passage apparenté à de la science-fiction dans Comment 
voyager avec un saumon d'Umberto Eco, auteur qu'elle assimile à la littérature légitime et qu'elle n'aurait jamais osé aborder par ailleurs (« Umberto Eco, c'est celui qui a écrit Le Pendule de Foucault, c'est genre une tête de la littérature. Moi, je m'attaque pas à ce genre de choses $»)$.

De même Olivier, issu d'une famille plus populaire (père chef mécanicien, mère aidesoignante), qui a suivi des études supérieures scientifiques, parvient par la sciencefiction à "se réconcilier avec la littérature classique ", à travers la lecture de l'Histoire comique des États et Empires de la Lune de Cyrano de Bergerac.

"Je suis pas du tout littérature classique, j'arrive pas du tout. [...] Sur internet je devais lire, peut-être un forum ou une page Wikipédia qui parlait de science-fiction, ou quelqu'un disait globalement ça c'est un des premiers bouquins de sciencefiction, je me suis dit "ah c'est trop bien j'ai envie de le lire"... [...] C'est ça, comme ça je pourrais dire, je lis de la littérature classique. Et finalement j'ai lu ben les deux, parce qu'il y a le premier où il va sur la lune et le deuxième où il va sur le soleil. Donc ceux-là j'ai lu les deux. Eh ben finalement ça m'a entre guillemets réconcilié avec la littérature classique. » Olivier, 26 ans, étudiant en préparation à l'agrégation de physique-chimie, doctorat d'astrophysique, père chef mécanicien, mère aidesoignante.

\section{Conclusion}

Malgré le défaut de légitimité dont souffrent les littératures de l'imaginaire dans leur ensemble, elles peuvent faire l'objet de modes de lecture savants, de réceptions légitimes, et constituer ainsi des ressources culturelles. De multiples stratégies de distinction sont pour cela mises en place par leurs lecteurs et lectrices; elles s'appuient sur une différenciation et une hiérarchisation des manières de lire, mais aussi des titres lus. En outre, le cas des littératures de l'imaginaire montre que les phénomènes de distinction ne sont pas l'apanage des classes supérieures. Dans cette enquête, le principe de distinction mis en évidence par Bourdieu n'est pas démenti par la montée de l'omnivorisme (Peterson 2004). Mais loin d'être seulement un moyen par lequel les classes supérieures s'opposent aux classes moyennes et les classes moyennes aux classes populaires, il est aussi perceptible parmi les individus que leur héritage ou leur formation classe parmi les moins dotés. Les études sur les styles de vie et pratiques culturelles des classes moyennes et populaires ne cessent en effet de rappeler l'hétérogénéité de ces dernières (Bernard 2013 ; Cartier, Coutant, Siblot, Masclet 2008 ; Mauger 2013). De la même manière, la lecture de littératures de l'imaginaire met en évidence la possibilité de "micro-distinctions ", même chez les individus moins bien pourvus en capitaux culturels, qui trouvent alors dans leurs pratiques lectorales des ressources symboliques, illustrant ainsi la persistance des logiques de distinction et des hiérarchies culturelles de légitimité, même au sein d'univers culturels éclectiques. 


\section{BIBLIOGRAPHIE}

BERNARD Lise (2013). « Réflexions sur la "petite bourgeoisie nouvelle” dans les années 2000 ». In COUlANGEON Philippe \& DUVAL Julien (dir.). Trente ans après « La Distinction » de Pierre Bourdieu. Paris, La Découverte : 266-277.

BOURDIEU Pierre (1979). La Distinction. Critique sociale du jugement. Paris, Minuit.

BOURDIEU Pierre (1991). « Le Champ littéraire ». Actes de la recherche en sciences sociales, 89 : 3-46.

BUISSON-FENET Hélène \& LANDRIER Séverine (2008). « Être ou pas ? Discrimination positive et révélation du rapport au savoir. Le cas d'une "prépa ZEP” de province ». Éducation et sociétés, 21 : 67-80.

CARTIER Marie, COUTANT Isabelle, SIBLOT Yasmine, MASCLET Olivier (2008). La France des « petitsmoyens ». Enquête sur la banlieue pavillonnaire. Paris, La Découverte.

CHARPENTIER Isabelle (2006). « Pour une sociologie de la réception et des publics ». In CHARPENTIER Isabelle (dir.). Comment sont reçues les œuvres? Actualités des recherches en sociologie de la réception. Paris, Créaphis : 5-22.

CHARTIER Roger (1985). Pratiques de la lecture. Marseille, Payot \& Rivages.

COLLOVALD Annie \& NeVeu Éric (2004). Lire le noir. Enquête sur les lecteurs de récits policiers. Paris, Éditions de la BPI-Centre Pompidou.

COULANGEON Philippe (2003). « La Stratification sociale des goûts musicaux. Le modèle de la légitimité culturelle en question ». Revue française de sociologie, 44(1) : 3-33.

COULANGEON Philippe (2004). «Classes sociales, pratiques culturelles et styles de vie. Le modèle de la distinction est-il (vraiment) obsolète ?». Sociologie et sociétés, 36(1) : 59-85.

DÉTREZ Christine \& VANHÉE Olivier (2012). Les Mangados. Lire des mangas à l'adolescence. Paris, Éditions de la BPI-Centre Pompidou.

DONNAT Olivier (2009). Les Pratiques culturelles des Français à l'ère numérique. Paris, La Découverte. GLEVAREC Hervé \& PINET Michel (2009). « La “Tablature” des goûts musicaux. Un modèle de structuration des préférences et des jugements ». Revue française de sociologie, 50(3) : 599-640. HOMMEL Elodie (2017). Lectures de science-fiction et fantasy. Enquête sociologique sur les réceptions et appropriations des littératures de l'imaginaire. Thèse de doctorat en sociologie. Lyon, Université de Lyon.

JAKMAKEJIAN Aurélia (2001). « Science-fiction : la ruée vers la fantasy ». Livres Hebdo, 427 : 65-70. KLEIN Gérard (1977). « Le Procès en dissolution de la science-fiction, intenté par les agents de la culture dominante ». Europe, 580-581.

LAHIRE Bernard (1993). La Raison des plus faibles. Rapport au travail, écritures domestiques et lectures en milieux populaires. Lille, Presses universitaires de Lille.

LAHIRE Bernard (2004). La Culture des individus. Dissonances culturelles et distinction de soi. Paris, La Découverte.

LE BART Christian (2004). «Stratégies identitaires de fans ». Revue française de sociologie, 45(2): 283-306. 
LE GUERN Philippe (2002). Les Cultes médiatiques. Culture fan et œuvres cultes. Rennes, Presses universitaires de Rennes.

MARIDET Morgane (2016). La Khâgne, un nouveau chapitre. Élaborations et reconstructions du rapport à la lecture des étudiants en classe préparatoire littéraire. Thèse de doctorat en sociologie. Paris, Université Sorbonne nouvelle.

MAUGER Gérard, POLIAK Claude, PUDAL Bernard (1999). Histoires de lecteurs. Paris, Nathan.

MAUGER Gérard (2013). « Bourdieu et les classes populaires. L'ambivalence des cultures

dominées ». In COULANGEON Philippe \& DUVAL Julien (dir.). Trente ans après « La Distinction » de Pierre Bourdieu. Paris, La Découverte : 243-254.

PETERSON Richard (2004). « Le Passage à des goûts omnivores. Notions, faits, perspectives ». Traduit de l'anglais par Suzanne Mineau. Sociologie et sociétés, 36(1) : 145-164.

PETIT Michèle (2002). Éloge de la lecture. La construction de soi. Paris, Belin.

RENARD Fanny (2011). Les Lycéens et la lecture. Entre habitudes et sollicitations. Rennes, Presses universitaires de Rennes.

SUVIN Darko (1977). Pour une poétique de la science-fiction. Études en théorie et en histoire d'un genre littéraire. Québec, Presses de l'université du Québec.

TORRES Anita (1997). La Science-fiction française. Auteurs et amateurs d'un genre littéraire. Paris, L'Harmattan.

\section{NOTES}

1. L'utilisation du sigle "SF" (science-fiction) constitue un marqueur de familiarité avec le genre, voire de connivence avec le destinataire. Il est donc repris tel quel dans les citations quand il a été employé ainsi par les personnes interrogées.

2. Deux ou trois ans d'études après le baccalauréat, qui est le diplôme de fin d'études secondaires en France.

3. La terminale est la dernière année d'études secondaires en France. L'appellation «niveau terminale » s'applique aux individus qui ont arrêté leur scolarité avant de passer le baccalauréat, c'est-à-dire leur diplôme de fin d'études secondaires.

4. Au moment de l'entretien, Marc semble être dans une situation conflictuelle avec sa famille, à laquelle il ne souhaite pas demander de l'aide et dont il évite de parler. Nous ne disposons donc pas des éléments nécessaires pour étayer plus en détails cette hypothèse.

5. «Les dernières polémiques à propos des programmes de télévision datent du lancement de la première édition du jeu de téléréalité Loft Story au début des années 2000 ; depuis cette date, les discours sur les effets de violence des images sur les enfants et les adolescents ou sur les dangers de l'addiction se sont déplacés vers les jeux vidéo et l'internet » (Donnat 2009 : 73).

6. Les classes préparatoires sont un ensemble de filières d'enseignement supérieur sélectives qui préparent aux concours d'entrées des grandes écoles françaises.

7. L'agrégation est le concours le plus prestigieux pour le recrutement des professeur·e's de l'enseignement secondaire français.

8. «Ça aurait été plus dans une géographie de représentation de l'espace, et moi j'étais plus dans une géographie plus quantitative [...] Donc j'étais pas dans le bon milieu en géographie».

9. L'opposition production restreinte/production commerciale recoupe par ailleurs en partie les oppositions en termes de définition, puisque les défenseurs d'une science-fiction «de projection » (aussi appelée fiction spéculative) revendiquent également sa qualité littéraire. 
10. Cette représentation graphique s'appuie sur le modèle d'analyse utilisé par Annie Collovald et Erik Neveu (Collovald \& Neveu 2004), en prenant en compte le capital économique et le capital symbolique des maisons d'édition et collections présentées, mais aussi sur le travail, réactualisé, d'Anita Torres (Torres 1997). Y sont ainsi indiquées celles qui publient uniquement de la sciencefiction " pure » (comprendre "scientifique », au sens de Gérard Klein), et celles qui se consacrent exclusivement à la fantasy, la majorité des éditeurs publiant aussi bien l'une que l'autre (pour plus de détails, voir Hommel 2017 : 55-75).

11. Cet anglicisme est employé par plusieurs enquêté'e-s, au sens de conformiste, sans originalité.

12. En raison du caractère imaginaire $d u$ genre, ses lecteurs et lectrices sont en effet surexposée's au soupçon de ne pas parvenir à distinguer la réalité de la fiction, ou de chercher à fuir le réel.

13. Littéralement "littérature pour poulette ", la chick-lit désigne de façon péjorative une production romanesque, souvent sentimentale, à destination de jeunes femmes. Elle peut comprendre aussi bien des romans réalistes que des littératures de l'imaginaire.

14. Bien que certain'e's enquêtée's présentent des pratiques perçues comme moins légitimes (collection, cosplay), celles-ci coexistent dans notre échantillon avec des modes de lectures qui impliquent une certaine réflexivité (ressource pour analyser le monde qui les entoure, aide à la construction de soi, parmi d'autres usages «éthico-pratiques »), même en l'absence de lectures « savantes » à proprement parler.

15. Le BTS (Brevet de technicien supérieur) est un diplôme d'études supérieures qui se prépare en deux ans après les études secondaires.

16. La maîtrise est l'ancien nom du Master 1 en France, diplôme universitaire correspondant à quatre ans d'étude après les études secondaires.

17. L'étude des sociabilités lectorales autour des littératures de l'imaginaire menée par ailleurs (Hommel 2017 : 315-346) a montré que ces lectures étaient particulièrement susceptibles de donner lieu à des discussions, au sein de groupes d'amateurs et amatrices, y compris en ligne, mais aussi avec des non-lecteur-rices du genre. Ces échanges contribuent à la formalisation d'une réflexion critique sur les titres lus mais aussi à la transmission de normes de «bon goût » en la matière. Les lecteurs et lectrices les plus assidu·e's font généralement figure d' "expert·e·s ", prodiguant conseils de lecture et avis critiques, dans le cadre de ces sociabilités.

18. Bien que publié en 1954-1955, Le Seigneur des anneaux inclut des passages en vieil anglais, utilisé pour transcrire la langue de la contrée fictive du Rohan.

19. Langues, littératures et civilisations étrangères.

20. Les titres publiés par Milady concentrent une bonne partie de ces préjugés, en particulier ceux associés à la bit-lit (littérature sentimentale mettant en scène vampires et loups-garous).

21. Le Silmarillion est une œuvre complémentaire au Seigneur des anneaux et au Hobbit, qui retrace la genèse et la mythologie de la Terre du Milieu.

22. Le DUT (diplôme universitaire de technologie) est un diplôme universitaire correspondant à deux ans d'étude après les études secondaires.

23. Le Deug (diplôme d'études universitaires générales) est un ancien diplôme universitaire correspondant à deux ans d'étude après les études secondaires.

24. Le Master 1 correspond à quatre années d'étude après les études secondaires.

25. Surtout des policiers, concernant la mère de Marc, "tout Stephen King " et " des romans contemporains », pour la mère de Benjamin, abonnée à France Loisirs. 


\section{RÉSUMÉS}

Les littératures de l'imaginaire, catégorie éditoriale qui regroupe science-fiction et fantasy, présentent des niveaux de légitimité variables selon les titres. Cet article propose une analyse des enjeux lectoraux liés à la légitimité ambiguë de ces productions chez les lecteurs et lectrices, en décrivant comment cette lecture soupçonnée d'illégitimité peut se transformer en une ressource sociale et culturelle. Après avoir évoqué les jugements auxquels font face les lecteurs et lectrices de science-fiction et fantasy, il montre comment la hiérarchie de légitimité interne au genre rend possible la mise en place de stratégies de distinction chez les individus à fort capital culturel, mais pas uniquement, à travers le choix des titres lus et les modes de lecture adoptés.

The legitimacy of speculative fiction - a broad fiction category that includes science fiction and fantasy - varies according to the choice of texts. This article analyses what is at stake when reading literary genres with ambiguous legitimacy, by describing how this supposed illegitimate reading can be transformed into a social and cultural resource. After discussing the prejudices that science fiction and fantasy readers face, it shows how the genre's internal legitimacy hierarchy allows for strategies of distinction amongst, not only individuals with a strong cultural capital, but others as well, through their choice of books and adopted reading methods.

\section{AUTEUR}

\section{ÉLODIE HOMMEL}

Université Paul Valéry (Montpellier 3)/Centre Max Weber 